\title{
Age of onset should guide germline mutation testing
}

Patients who present with renal cell carcinoma (RCC) at the age of 46 years or younger should be referred for germline mutation testing for hereditary disease-even in the absence of clinical manifestations-according to a team of researchers led by Marston Linehan at the US National Cancer Institute (NCI).

Linehan and his colleagues have a long history of managing patients with kidney cancer, especially those with hereditary RCC syndromes. Unfortunately, such syndromes can be challenging to diagnose if the clinical features are subtle and there is no family history; earlier diagnosis and treatment is likely to improve outcomes.

Given the link between hereditary cancers and early onset, and the current lack of guidelines for the selection of patients with kidney cancer for germline mutation testing, Linehan and colleagues sought to find an age threshold that they could use to guide this decision. They analysed data from 106,224 patients with kidney cancer in the SEER database to elucidate the age of onset in the general population, then compared the age distribution with a cohort of 608 patients with known hereditary RCC syndromes from their own institution.

\section{4 ...earlier diagnosis and treatment is likely to improve outcomes 77}

Age of onset was significantly lower for hereditary disease; the median age of RCC onset in SEER patients was 64 years compared with 37 years in the NCI cohort. The investigators then performed statistical modelling to assess whether a specific age threshold could be used to identify hereditary cancers in the general population. They found that using the $10^{\text {th }}$ percentile-corresponding to 46 years-provided the best combination of sensitivity (70\%), specificity (90\%) and number needed to treat to find one case of hereditary RCC (7-28 individuals).

Although this is an important first step in defining early-onset disease, it is likely that the age threshold will differ between syndromes, given the differences noted in median age of onset (ranging from 35 years in patients with von Hippel-Lindau syndrome to 50 years in those with Birt-Hogg-Dubé syndrome). Furthermore, patients with hereditary papillary RCC $(n=25)$ and succinate dehydrogenase subunit B RCC $(n=14)$ are under-represented in the NCI cohort.

"Although we have identified a number of genes (not all of them germline) that are associated with the development of early-onset kidney cancer, we still see a number of patients with early-onset kidney cancer for which we do not have a gene," says Linehan. "In the future, we will conduct extensive genomic analysis to identify novel genes associated with the development of early-onset kidney cancer."

Sarah Payton

Original article Schuch, B. et al. Defining early-onset kidney cancer: implications for germline and somatic mutation testing and clinical management. J. Clin. Oncol. doi:10.1200/JC0.2013.50.81925 